http://dx.doi.org/10.18778/2196-8403.2019.01

\title{
Der performative Zauber der Sirenen? ADORNOS negative Dialektik und das deutschsprachige Gegenwartstheater
}

Wieso flüchtete ADORNo, der Philosoph der Student*innenrevolte, der Fürsprecher der Avantgarde von Schönberg bis Beckett, im April 1969 vor der Performance protestierender Studentinnen mit nackten Brüsten? Der Beitrag erörtert Verbindungen von ADORNOS Dialektik der Aufklärung, Negativer Dialektik und Ästhetischer Theorie zur Ästhetik des heutigen performativen oder postdramatischen Theaters. Dennoch blieb für ADORNO das Theater ein Ort der Kunst, verwirklichte sich die Revolution nicht in der performativen Verweigerung von Sinn, im grausamen Realen der ,Sirenen' und der Dekonstruktion, sondern in einem Werk, das das Unversöhnliche darzustellen und so zu versöhnen habe.

The Performative Magic of Sirens? Adorno's Negative Dialectics and Germanspeaking Contemporary Theater

Why, in April 1969, did ADORNO, the philosopher of the student revolt, the advocate of the avant-garde from Schoenberg to Beckett, flee when he was faced with the performative actions of protesting students with naked breasts? The article discusses connections between ADORNO's Dialectics of Enlightenment, Negative Dialectics and Aesthetic Theory and the aesthetics of today's performative and post-dramatic theatre. For ADORNO, theatre remained a place of art, and the revolution did not come to fruition in the performative denial of meaning, in the cruel reality of "sirens", and in deconstruction, but in the work of art itself, that is supposed to represent the irreconcilable and thus to lead to reconciliation.

Performatywny czar syren? Negatywna dialektyka ADORNo a współczesny teatr niemieckojęzyczny

Dlaczego ADORNO, filozof rewolty studenckiej, zwolennik awangardy od Schönberga po Becketta uciekł w kwietniu 1969 roku przed performatywnym protestem studentek, 
obnażających nagie biusty? Artykuł wyjaśnia związki między Dialektyka oświecenia, Dialektyka negatywna i Teoria estetyczna z estetyką dzisiejszego teatru performatywnego i postdramatycznego. Mimo wszystko teatr pozostał dla ADORNA miejscem sztuki, a rewolucja dokonała się nie w performatywnej negacji sensu, w okrutnej realności ,syren' i dekonstrukcji, lecz w dziele, które przedstawiając to, co nie do pogodzenia, prowadzić ma do ugody.

\section{Adornos Flucht vor den Sirenen}

Am 22. April 1969 betrat der 65-jährige THEODOR W. ADORNO den Hörsaal VI der Goethe-Universität Frankfurt, um wie gewöhnlich seine Vorlesung zu halten. Es waren keine ruhigen akademischen Zeiten, die studentischen Unruhen hatten bereits mehrere Höhepunkte hinter sich, der Vietnamkrieg tobte, die Linke radikalisierte sich zunehmend. Dabei galt ADORNO bis kurz vorher noch als revolutionärer Vordenker, seine Vorlesungen waren außerordentlich gut besucht. Doch seit er im Januar 1969 sein von Studierenden besetztes Institut von der Polizei hatte räumen lassen, wurde ihm Verrat vorgeworfen. ADORNO konnte seine Vorlesung aufgrund der Unruhe nicht beginnen, an der Tafel musste er lesen: „Wer nur den lieben ADORNO lässt walten, der wird den Kapitalismus sein Leben lang behalten.“ ADORNO formulierte ein Ultimatum: „Ich gebe Ihnen fünf Minuten Zeit. Entscheiden Sie, ob meine Vorlesung stattfinden soll oder nicht" (LEMHÖFER 2008). Plötzlich näherten sich drei Studentinnen in Lederjacken, öffneten diese und rückten dem Gelehrten mit nackten Brüsten buchstäblich auf den Leib, versuchten, ihn mit Blumen zu bewerfen und zu küssen. ADORNO reagierte abwehrend, verließ fluchtartig den Saal. Wieso handelte ADORNO, der in dieser Zeit eine Affäre mit einer weit Jüngeren hatte, auch einem Besuch im Bordell nicht abgeneigt war, so unsouverän? Ende der 1960er-Jahre war man doch gerade im universitären und kulturellen Milieu einiges gewohnt. In Frankfurt sah man eine als Skandal empfundene Publikumsbeschimpfung des jungen Peter Handke, also eines Autorenkollegen bei Suhrkamp. ${ }^{1}$ Man hörte von Allan Kaprows Happenings und den Fluxusaktionen

Diese sogenannte Suhrkampkultur verstand sich als zeitgenössische Speerspitze des intellektuellen Diskurses. Sie ist ein bis heute noch nicht erschöpfend untersuchtes Phänomen nach 1945, das parallel mit der Dominanz der Gruppe $47 \mathrm{im}$ intellektuellen Leben, einhergehend mit der Ausweitung der Bildungskultur und des Fächerkanons in den 1960er-Jahren in Westdeutschland (in Ostdeutschland reüssierte eher die Belletristik) virulent wurde: Theorie wurde ein Leitmedium, ähnlich wie noch das Theater in der damaligen Zeit. Literatur, Theorie und Theater 
von Nam June Paik oder Joseph Beuys. Nacktheit war in Deutschland schon seit der Jahrhundertwende und der Weimarer Republik kein Aufregerthema mehr, nach Lebensreformbewegung, FKK-Kultur und Nackttanz von Isadora Duncan bis Josephine Baker (vgl. TRAUB 2010). Das Orgien Mysterien Theater Hermann Nitschs, der Wiener Aktionismus, das Living Theatre und die Performance Group Richard Schechners schufen über die Nacktheit eine ritualisierte Form des Protests; öffentlich provozierten Studentinnen auf Demonstrationen und revolutionär gestimmte Linke in Gerichtssälen ebenfalls mit nackten Brüsten. Diese Tradition einer Performance der Nacktheit setzt sich bis heute fort, man denke nur an die ukrainischen feministischen Performerinnen von Femen, die mit nackten Brüsten gegen Sextourismus agitieren. 1969 regte sich ADORNO jedenfalls auf, mehr als ihm gut zu tun schien - später behaupteten einige, dass dieses ,Busenattentat' mitschuldig sei, dass er noch im Sommer des gleichen Jahres bei einer Bergtour an einem Herzinfarkt starb. Mutmaßlich waren mehr seine von ihm wenig beachtete Diabetes, die Sommerhitze, die ungewohnte Anstrengung im Schweizer Hochland und das bereits vorgerückte Alter die wahre Ursache. Die Frage, die sich theater- und kunstwissenschaftlich stellt, ist aber eine andere: Wieso wehrte ADORNO, immerhin ein Fürsprecher der Avantgarde, von Arnold Schönbergs Zwölftonkompositionen bis zu Samuel Becketts absurdem Theater, diese doch eher harmlose Performance der Studentinnen so ab? ${ }^{2}$ Oder anders formuliert: Verkörperte bzw. performte ADORNO im ,Busenattentat" nicht paradigmatisch die mit MAX HORKHEIMER in der Dialektik der Aufklärung dargestellte Urszene des modernen Menschen, des Odysseus vor den Sirenen?

\section{Die performativen Sirenen der Counterculture der 1960er- Jahre}

Die Sirenen waren ein wichtiges Motiv der Counterculture. Das Jahr 1969 brachte bereits etwas zu einem Abschluss, was als vorstellungsweltliche Veränderung in den 1960er-Jahren begann. Drei theoretische Texte stellten eine

wurden unter dem Dach der innovativen Taschenbuchreihe edition suhrkamp (nicht ohne sozialisierenden Anspruch: Theorie im Taschenbuch für alle) vereinigt, vertreten etwa durch ADORNOS, Bert Brechts, Peter Handkes und Niklas Luhmanns Texte.

2 Immerhin stand er zumindest schreibend sogar dem Surrealismus nicht ablehnend gegenüber, wie seine Traumprotokolle beweisen (vgl. ADORNO 2005). 
besonders revolutionäre Lektüre dar: Herbert Marcuses One-Dimensional Man von 1964, Michel Foucaults Les mots et les choses. Une archéologie des sciences humaines und ADORNOS Negative Dialektik, beide von 1966. Zur gleichen Zeit veränderte sich das Theater in Westdeutschland grundlegend. Peter Zadek, Peter Stein und Claus Peymann erkämpften sich sukzessive den Freiraum für das zukünftige Regietheater und ihre weitere Karriere, die Neoavantgarde (von John Cage bis zu den Situationisten) überbrückte die Kluft zwischen bildender Kunst, Performance Art und Theater. Jerzy Grotowski, Tadeusz Kantor und Peter Brook erarbeiteten ein völlig neues Theaterverständnis und der us-amerikanische Theaterwissenschaftler Richard Schechner prägte 1966 Begriff und Theorie der Ästhetik des ,Postdramatic Theatre‘ (vgl. SCHECHNER 1966). Schechner fasste die experimentellen Ästhetiken der 1960er- und 1970er-Jahre in seiner Entgegensetzung von einem ,progressiven“ ritualähnlichen Theater der Wirksamkeit und einem ,rückständigen“ Theater der Unterhaltung systematisch und historisch so folgenreich (vgl. SCHECHNER 1973), dass auf der Basis seiner Überlegungen und Schemata der polnische und später in den USA lehrende Theaterwissenschaftler Andrzej Wirth in den frühen 1980er-Jahren das Gießener Institut für Angewandte Theaterwissenschaft gründen konnte (vgl. WIRTH 1985). Die Gießener Schule des postdramatischen Theaters wurde so - wenn auch mehr theoretisch als tatsächlich, denn performative Ästhetiken waren gerade in der freien Szene, von Fassbinder bis Sagerer, schon weit vor der Gründung Gießens dominant - im deutschsprachigen Raum zum Hort des performativen Theaters und schuf sukzessive an öffentlich geförderten traditionellen Bühnen für die immer mehr reüssierende postdramatische Ästhetik spürbaren Rückenwind. Die heute meistzitierten Standortbestimmungen des postdramatischen Theaters sind die des ehemaligen Assistenten Andrzej Wirths, Hans-Thies Lehmann. Er griff Überlegungen Schechners und Wirths auf und machte sie in den 1990er-Jahren für das Politische im Sinne einer negativen Dialektik ADORNOS, aber auch der Dekonstruktion aus französischneostrukturalistischer Perspektive im Theater fruchtbar. Der Andere (als Existenz, zu Begegnender, Anwesender etc.) sei, so Lehmann, ständig verstellt, durch das „,sozio-symbolische Gesetz“ (LEHMANN 1999:457). Diese Gestalt und Gestaltung des Anderen zeichnet ein Bild des Anderen, das den Vorteilen aus der symbolischen Ordnung und keineswegs der unendlich komplexe Andersheit des Anderen entspricht. Das sozio-symbolische Gesetz wäre dabei nach Lehmann „das gemeinsame Maß, das Politische, der Bereich von dessen Bestätigung, Bekräftigung, Sicherung, Anpassung an den wandelbaren Lauf der Dinge, Abschaffung oder Modifizierung" (LEHMANN 1999:457). Dementsprechend existiere 
eine unaufschiebbare Kluft schon im Politischen, das die Regel gibt, und der Kunst, die, sagen wir einfach, immer die Ausnahme ist: Ausnahme zur Regel, Affirmation des Nichtregelhaften sogar noch in der Regel selbst. Theater als ästhetisches Verhalten [sei...] undenkbar ohne das Moment der Übertretung der Vorschrift, der Überschreitung. (LEHMANN 1999:457)

Diese Überschreitung kritisiert, stellt in Frage, dekonstruiert oder löscht gar die Konstituenten des Dramas (Figur, Handlung, Ort, Zeit und Dialog) aus. Sie verhindert die Repräsentation der Außenwelt durch das Bühnenspiel und subvertiert die klare Unterscheidung zwischen Schauspielen (Rollenspiel) und Alltagsrolle (im soziologischen Sinn) bzw. zwischen Schauspielen und Performen.

Man könnte nun zwei Thesen formulieren: Erstens könnte man annehmen, dass ADORNOS theoretische Überlegungen unter anderem zu einer der Grundlagen jenes performativen Theaterverständnisses werden, das in den 1960er-Jahren zu einer der wichtigsten Ästhetiken des deutschsprachigen Theaters wurde. Zweitens könnte die von Lehmann geforderte Überschreitung als Annäherung an die Sirenen, also die bedeutungsverweigernde Präsenz des Performativen verstanden werden, die ADORNO - zumindest was seine Person betrifft - nicht mitmachen wollte.

\section{Odysseus und die Sirenen (Dialektik der Aufklärung)}

Das Theater bzw. jede darstellende Dramaturgie allgemein gründete in der griechischen Polis. Meist waren Mythen die Basis des Theaters seit dem 6. Jahrhundert v. Chr. im Athener Dionysostheater, auch wenn die zunehmend dialogischen Aufführungen die Mythen im Theaterspiel bereits mehr oder weniger in ihrer absoluten Gültigkeit relativierten. Homers Ilias und Odyssee, die älter als das Theater sind und wahrscheinlich aus dem 8. oder 7. Jahrhundert vor Christus stammen, sind ebenfalls Zeugnisse mythischer Vorstellungen zu Beginn der griechischen Kultur. Sie können zugleich als Quellen gedeutet werden, in denen sich der Mensch in seinem verändernden Verhältnis zu sich und der Umwelt zum Ausdruck bringt. 1944 bzw. 1947 veröffentlichten HORKHEIMER und ADORNO (und, wie man heute weiß, Gretel Adorno, die die Texte wohl nicht nur redigiert, sondern auch teilweise mitverfasst hat) die Dialektik der Aufklärung, die für die Counterculture der 1960er-Jahre so wichtig wurde, dass sie unter den revoltierenden Studierenden als Raubdruck kursierte. Eine der zentralen Thesen ist, dass man nach den Verbrechen der ersten Hälfte des 20. Jahrhunderts, die paradoxerweise nach der Aufklärung des Menschen im bürgerlichen Zeitalter erfolgten, das Verhältnis zu Mythen und Aufklärung anders verstehen 
müsse: „Wie die Mythen schon Aufklärung vollziehen, so verstrickt Aufklärung mit jedem ihrer Schritte tiefer sich in Mythologie" (HORKHEIMER / ADORNO 1968:22). Damit könne man die Abenteuer des Odysseus als Bericht einer frühen Aufklärung des ,Ichs' verstehen. Zugleich seien sozusagen spiegelbildlich heutige Strukturen einer aufgeklärten Kultur und Gesellschaft als von Mythologie durchzogen zu interpretieren. Wenn man Aufklärung mit bürgerlichem Theater, bürgerlicher Dramatik und bürgerlicher Dramaturgie gleichsetzt, werden HORKHEIMERS und ADORNOS Thesen für die Theater- und Kulturwissenschaft interessant. Odysseus geht, wie es sich für einen aufgeklärten Helden gehört, informiert, überlegt und intelligent in die Begegnung mit den Sirenen. Gleichwohl ist er noch auf die verführerische Zauberin Kirke angewiesen ist, die ihn warnt:

Erstlich erreichet dein Schiff die Sirenen; diese bezaubern / Alle sterblichen Menschen, wer ihre Wohnung berühret. / Welcher mit törichtem Herzen hinanfährt und der Sirenen Stimme lauscht, dem wird zu Hause nimmer die Gattin / Und unmündige Kinder mit freudigem Gruße begegnen; / Denn es bezaubert ihn der helle Gesang der Sirenen, / Die auf der Wiese sitzen, von aufgehäuftem Gebeine / Modernder Menschen umringt und ausgetrockneten Häuten. (HOMER 1977:171f., zwölfter Gesang, Zeilen 39ff.)

Die Sirenen versprechen außerordentliches Vergnügen und allumfassendes Wissen. In den 1940er-Jahren seien, so HORKHEIMER und ADORNO, die Sirenen noch als Mächte der „Auflösung“, als elementare Bedrohung der herrschenden „patriarchale[n] Ordnung, die das Leben eines jeden nur gegen sein volles Maß an Zeit zurückgibt", zu begreifen (HORKHEIMER / ADORNO 1968:47). Die Bedrohung der Sirenen liege darin, dass sie das lockende Negativbild sich aufklärender Menschheit wären, für die Odysseus steht. Nicht einmal der Listigste könne sich daher „entziehen“, denn

Furchtbares hat die Menschheit sich antun müssen, bis das Selbst, der identische, der zweckgerichtete, männliche Charakter des Menschen geschaffen war, und etwas wird davon noch in jeder Kindheit wiederholt. Die Anstrengung, das Ich zusammenzuhalten, haftet dem Ich auf allen Stufen an, und stets war die Lockung, es zu verlieren, mit der blinden Entschlossenheit zu seiner Erhaltung gepaart. (HORKHEIMER / ADORNO 1968:47)

Dialektisch gesehen, so HORKHEIMER und ADORNO, beruhe die Konstruktion der modernen Gesellschaft auf dem Tabu der rauschhaften Präsenz des Lebens selbst. Der „,narkotische Rausch, der für die Euphorie, in der das Selbst suspendiert ist, mit todähnlichem Schlaf büßen“ ließe, wäre „eine der ältesten gesellschaftlichen Veranstaltungen, die zwischen Selbsterhaltung und -vernichtung vermittelt, ein Versuch des Selbst, sich selber zu überleben“ (HORKHEIMER / ADORNO 
1968:47). Die Sirenen seien somit das Andere im dramatisch-stabilen Selbst der eigenen Figur: „Die Angst, sich selbst zu verlieren, und mit dem Selbst die Grenze zwischen sich und anderem Leben aufzuheben, die Scheu vor Tod und Destruktion, ist einem Glücksversprechen verschwistert, von dem in jedem Augenblick die Zivilisation bedroht war." Moderne, arbeitsteilige Gesellschaften funktionierten wie Odysseus auf dem Weg ,,von Gehorsam und Arbeit, über dem Erfüllung immerwährend bloß als Schein, als entmachtete Schönheit lauert“ (HORKHEIMER / ADORNO 1968:47).

Anhand der Geschichte der Begegnung mit den Sirenen ließen sich marxistisch klassenbewusst zwei gesellschaftlich differente Möglichkeiten des gesellschaftserhaltenden Umgangs mit den Verlockungen zur Erfüllung nachzeichnen: Die für die unteren Klassen schreibt Odysseus seinen ihm untergebenen Begleitern vor. Er

verstopft ihnen die Ohren mit Wachs, und sie müssen aus Leibeskräften rudern. Wer bestehen will, darf nicht auf die Lockungen des Unwiederbringlichen hören, und er vermag es nur, indem er nicht zu hören vermag. Dafür hat die Gesellschaft stets gesorgt. Frisch und konzentriert müssen die Arbeitenden nach vorwärts blicken, und liegenlassen, was zur Seite liegt. Den Trieb, der zur Ablenkung drängt, müssen sie verbissen in zusätzlicher Anstrengung sublimieren. So werden sie praktisch. (HORKHEIMER / ADORNO 1968:48)

Die herrschenden Klassen hingegen, für die die Figur des Helden Odysseus steht, die Grundherren, die andere für sich arbeiten lassen, später die Bürger, haben die Möglichkeit einer alternativen Begegnung mit den Verlockungen: Odysseus „hört, aber ohnmächtig an den Mast gebunden, und je größer die Lockung wird, umso stärker läßt er sich fesseln, so wie nachmals die Bürger auch sich selber das Glück um so hartnäckiger verweigerten, je näher es ihnen mit dem Anwachsen der eigenen Macht rückte“ (HORKHEIMER / ADORNO 1968:48). Klassendifferent bleibt ein Missverstehen; während die Gefährten nur die Gefahr bemerken, erlebt der Bürger Odysseus die umwerfende Schönheit der Sirenen: „Die Bande, mit denen“ sich Odysseus

unwiderruflich an die Praxis gefesselt hat, halten zugleich die Sirenen aus der Praxis fern: ihre Lockung wird zum bloßen Gegenstand der Kontemplation neutralisiert, zur Kunst. Der Gefesselte wohnt einem Konzert bei, reglos lauschend wie später die Konzertbesucher, und sein begeisterter Ruf nach Befreiung verhallt schon im Applaus. (HORKHEIMER / ADORNO 1968:48)

Auf das heutige Theater übertragen geht es hier um die Definition der ästhetischen Grenze zwischen Kunstraum und Realraum. In den Mittelpunkt rückt der Eindruck auf die Zuschauenden, das heißt jenes Phänomen, das Friedrich 
Nietzsche mit Blick auf Richard Wagners Musikdramen als das Dionysische bezeichnete (vgl. NIETZSCHE 1988:24). Von Nietzsche mit Blick auf Wagner mutmaßlich im Rahmen einer Illusionsästhetik gehalten vorgestellt, in der nur die Musik in den Zuschauer*innenbereich übergreift, radikalisiert die Avantgarde des 20. Jahrhunderts Nietzsches Überlegungen. Damit gewinnen die Sirenen im Theater immer mehr Aktionsradius, die Kraft der Kunst überschreitet in der Avantgarde zumindest programmatisch die Grenze zwischen Kunst und Leben (vgl. BÜRGER 1974; MENKE 2013). Sirenen symbolisieren also die Gefährdungen (neo)avantgardistischer Grenzüberschreitungen, von den Futurist*innen, Dadaist*innen und Surrealist*innen, von Antonin Artauds Theater der Grausamkeit bis hin zu Richard Schechners Environmental oder Postdramatic Theatre bzw. gegenwärtigem performativen Theater.

Diesem durch die Sirenen bewirkten, heute noch spürbaren (wenn auch vielleicht nicht mehr so ernst genommenen) Provokationspotential stehen die verschiedensten Publikumserwartungen gegenüber. Die Grenzen des ,Erträglichen werden dabei weiterhin gerne ausgetestet oder neu gezogen, grundsätzlich immer in den auf den Ebenen Körperlichkeit, Ekel oder Sadomasochismus tabubrechenden Aktionen von Florentina Holzinger, oft in den schwer verständlichen ästhetischen Assoziationen von Sebastian Hartmann, zuweilen in den überkomplexen musikalisch-performativen Inszenierungen von Nicolas Stemann. Diesem mehr oder weniger ausgeprägten Provokationspotential schließen sich Fragen zum aktuellen Theaterverständnis an. Inwieweit und in welcher sublimierten Form lässt sich das bürgerliche Publikum von den Verlockungen der performativen Präsenz der Sirenen anziehen? Man denke nur an die umjubelte Salome in der surrealen Regie von Romeo Castellucci bei den Salzburger Festspielen, die sogar dem konservativen Opernpublikum zusagte; oder eine zumindest freundlich vom Publikum aufgenommene Produktion wie Christopher Rüpings Dionysos Stadt an den Münchner Kammerspielen im Vergleich zu dem von einigen Zuschauer*innen aggressiv beschimpften Lear / Die Polizisten von Sebastian Hartmann am Deutschen Theater Berlin.

\section{Energie der Sirenen, ästhetisches Vergnügen und Klassen- bewusstsein}

HORKHEIMER und ADORNO deuten bereits in Homers Odyssee die Begegnung mit den Sirenen als Hinweis darauf, dass den unteren Klassen der Kunstgenuss verweigert werde, da man ihnen die Ohren mit Wachs verstopft. Für das heutige 
Theater ginge es folglich darum, ob und inwieweit sogenannte bildungsferne Schichten überhaupt ins Theater gehen. Man könne, so HORKHEIMER und ADORNO, schon bei Homer, der früh in der Kulturentwicklung die „richtige Theorie“ enthielt, nachlesen, wie „Kunstgenuß und Handarbeit im Abschied von der Vorwelt auseinander“ träten: „Das Kulturgut steht zur kommandierten Arbeit in genauer Korrelation, und beide gründen im unentrinnbaren Zwang zur gesellschaftlichen Herrschaft über die Natur" (HORKHEIMER / ADORNO 1968:47f.). Die Sirenen wären als (zu wilde) Natur zu beherrschen. Dass die Natur dialektisch gesehen schon lange im Anthropozän wieder zurückschlägt, ist spätestens mit der Akzeptanz der Existenz des Klimawandels kein Geheimnis mehr. Dabei hätte heutiges Theater sich der Aufgabe zu stellen, Kritik und Lösungen im Engagement an die Frage nach der Gerechtigkeit in der gesellschaftlichen Struktur zu binden. ${ }^{3}$ Das Gesellschaftspolitische hängt dabei über das Erkenntnistheoretische mit detaillierteren Strukturen des Dramatischen zusammen. In seiner Negativen Dialektik von 1966 wechselt ADORNO vom Allgemeinen einer Herrschaft über die gefährliche Natur ins Besondere des philosophischerkenntnistheoretischen Details:

Indem Denken sich versenkt in das zunächst ihm Gegenüberstehende, den Begriff, und seines immanent antinomischen Charakters gewahr wird, hängt es der Idee von etwas nach, was jenseits des Widerspruchs wäre. Der Gegensatz des Denkens zu seinem Heterogenen reproduziert sich im Denken selbst als dessen immanenter Widerspruch. Reziproke Kritik von Allgemeinem und Besonderem, identifizierende Akte, die darüber urteilen, ob der Begriff dem Befaßten Gerechtigkeit widerfahren läßt, und ob das Besondere seinen Begriff auch erfüllt, sind das Medium des Denkens der Nichtidentiät von Besonderem und Begriff. (ADORNO 1970:147)

Denken und Sein können bei ADORNO begrifflich keine Identität mehr behaupten. Übertragen auf das Drama bzw. den Theatertext auf der Bühne könnte man anschließen: Damit käme jede dramatische Eindeutigkeit spätestens seit Euripides oder Aristoteles unter Verdacht; dies umso mehr in einer industriellökonomischen Moderne, die über den Tausch die größtmögliche Ähnlichkeit von Allem mit Allem erforderlich macht: ,Soll die Menschheit des Zwangs sich entledigen, der in Gestalt von Identifikation real ihr angetan wird“, so ADORNO in der Negativen Dialektik, so müsse

sie zugleich die Identität mit ihrem Begriff erlangen. Daran haben alle relevanten Kategorien teil. Das Tauschprinzip, die Reduktion menschlicher Arbeit auf den

3 Vgl. insbesondere die Diskussion, die Thomas PiketTys (2014) Untersuchung Capital in the Twenty-First Century seit ihrer Veröffentlichung ausgelöst hat und die seither anhält. 
abstrakten Allgemeinbegriff der durchschnittlichen Arbeitszeit, ist urverwandt mit dem Identifikationsprinzip. Am Tausch hat es sein gesellschaftliches Modell, und er wäre nicht ohne es; durch ihn werden nichtidentische Einzelwesen und Leistungen kommensurabel, identisch. Die Ausbreitung des Prinzips verhält die ganze Welt zum Identischen, zu Totalität. (Adorno 1970:147)

In diesem Sinne werden mit kritischem Blick auf populäre Dramaturgien, die im Rahmen eines totalisierenden Verblendungszusammenhangs funktionieren, selbstverständliche Identitäten von Subjekt und Objekt bzw. Begriff und Ding als Konstruktion entlarvt. Nicht zufälligerweise heißt ein Titel von René Pollesch Ich schau dir in die Augen, gesellschaftlicher Verblendungszusammenhang! (POLLESCH 2010). Ähnlichkeit und Identifikation, die den Kapitalismus und eine mehr oder weniger verdeckte Klassenstruktur nach ADORNO erst ermöglichten, sind dann im postdramatischen Theater der Gegenwart das, was auf der Ebene der Identitätszuweisung im mehr oder weniger Dramatischen unter Verdacht steht bzw. dekonstruiert wird. Theater ist nach Matthias Lilienthal ein sozialer Ort, während Theater für ADORNO letztendlich ein ästhetischer Ort, eine Institution der Kunst blieb. Am sozialen Ort Theater treffe man - so Daniel Wetzel von Rimini Protokoll, dessen Mitglieder in den letzten Jahren vor allem mit sogenannten Experten des Alltags - Objets trouvés der Liveart gearbeitet haben - live und in Realpräsenz denjenigen Anderen, der ,anderes macht" (RIMINI PROTOKOLL 2006). Spannung entstehe im Theater, weil wir nicht wissen, womit wir als den ,realen "Anderen „diese gemeinsame Zeit“ (RIMINI PROTOKOLL 2006) verbringen und wie nahe wir Menschen uns „im Vollbesitz unseres Bewusstseins, dass wir unseren eigenen Kopf haben" (RIMINI PROTOKOLL 2006), geraten könnten. Die Menschen müssten, so Wetzel, „,immer wieder zusammenkommen“, um sich ihrer „Unterschiede gewahr zu werden. Gelacht wird im Theater allerdings dann, wenn es Gemeinsamkeiten gibt" (RIMINI PROTOKOLL 2006). ${ }^{4}$ Diese Gemeinsamkeiten verweisen auf Ähnlichkeiten in der kulturellen Zwischenwelt, wie sie René Pollesch in seinen Theatertexten als Ausdruck bürgerlicher Zwangskultur diskursiv ausstellt und ADORNO als Teil des gesellschaftlichen Verblendungszusammenhangs kritisierte. Damit wird etwas postdramatisch aufgebrochen, was auf einer etwas anderen Ebene aber mit ähnlicher Zielsetzung schon HORKHEIMER und ADORNO in ihrer

4 Diese Gemeinsamkeiten können gerade in der Ausgrenzung des Anderen, Fremden und Unbekannten durch das (Ver-)Lachen durchaus gewalttätig werden, wie ADORNO anlässlich des Lachens in einem Film von Charlie Chaplin feststellt; es wäre ,nichts weniger als gut und revolutionär sondern des schlechtesten bürgerlichen Sadismus voll“" (ADORNO / BENJAMIN 1994:171). 
Dialektik der Aufklärung angesprochen hatten. Auf der einen Seite sei die bürgerliche Gesellschaft „,beherrscht vom Äquivalent. Sie macht Ungleichnamiges komparabel, indem sie es auf abstrakte Größen reduziert" (HORKHEIMER / ADORNO 1968:18). Auf der anderen Seite werde die Identität „,von allem mit allem“ damit bezahlt, dass „,nichts zugleich mit sich selber identisch sein darf“ (HORKHEIMER / ADORNO 1968:23). In der Nachfolge von ADORNOS ästhetischer Theorie ist ästhetisches Verstehen prekäres Verstehen. Kunst provoziere eher Fragen, als dass es Antworten gäbe, funktioniere, so Georg W. Betram, als „Zeichen, an deren sinnlich-materialer Gestalt wir Verständnisse entwickeln“ (BETRAM 2005:296ff.). Dies gilt auch für die Theaterkunst, die von den Sirenen energetisch eröffnete ontologische Differenz von Sein und Seiendem, die „Duplizität von Bedeutung und Materialität“ (MERSCH 2001:276) verdeutlicht die Differenz zwischen „Repräsentation und Präsenz“ (BOEHM 2003:207) bzw. die von Darstellendem und Dargestelltem. Wirkungen als sinnlich-materiale Momente einer Darstellung oder performativen Präsenz werden in der Rezeption in Beziehung gesetzt, es zeigen sich Strukturen, auf kleiner Ebene verbinden sich Mikro- und Makrostrukturen des Eigenen und des Anderen, letztlich der (Theater-)Welt. Dabei bleiben aufgrund der Kraft der Sirenen Brüche, Nahtstellen, herrscht plötzlich Miss- oder Unverständnis. Das interpretierende Verstehen des modernen Menschen Odysseus wird von den Sirenen bis an seine eigenen Grenzen geführt, verlockt und provoziert. Projiziert auf überzeugende Theaterkunst, garantieren die Sirenen, dass diese generell uneinordbar, überoder untercodiert, erhaben, ständig auf Entzug oder fragmentiert ist. Insbesondere die Bedeutung und der Gehalt performativer Aktionen ist an die einmalige, individuelle Gestalt des Präsentischen gebunden, an - so Martin Seel - „Ereignisse des Erscheinens“, denen „eine besondere Gegenwärtigkeit“ zukomme; deren Vorgang des Erscheinens sei ein performativer Prozess, durch „den sie etwas in seiner Gegenwärtigkeit zur Darbietung bringen“" (SEEL 2007:35). Je näher man in den Machtbereich der Sirenen gerät, umso mehr erlebe man, dass Kunstwerke „Wahrnehmungsereignisse einer besonderen Art“ seien, „eben weil sie Darbietungsereignisse einer besonderen Art sind“ (SEEL 2007:35). In dieser Tautologie präsentierten sich Theaterpräsenzen so wie die Sirenen des Odysseus, indem sie sich selbst in ihrem So-Sein, in ihrer einmaligen sinnlichen Erscheinung präsentierten. Diese Einmaligkeit wäre, bezogen auf ADORNOs Überlegungen, die Bedingung des Entzugs im Nicht-Identischen, sei es im Drama bzw. Theatertext, sei es im Theater. Doch wie Nietzsche wusste, dass das Dionysische nicht ohne das Apollinische zur Darstellung kommen kann, so konstatiert ADORNO in seiner Negativen Dialektik: „Kein Sein ohne Seiendes“ 
(ADORNO 1970:137). Und vor dem Hintergrund dieser dem Theater eigenen mimetischen Differenz von Darstellendem und Dargestelltem ${ }^{5}$ sollten Machtverhältnisse und zuweilen schwer zu erkennende Ungerechtigkeiten nicht ignoriert werden. Die Frage nach der Gerechtigkeit, nach der Existenz von Klassen (und nicht etwa nur Milieus oder Erlebnissen), werde im performativen Theater mehr oder weniger ignoriert, so Bernd Stegemann in seiner Kritik des Theaters und Thomas Ostermeier in seinem Plädoyer für einen sozialen Realismus (vgl. STEGEMANN 2015; OSTERMEIER 2009).

\section{5. Ästhetische Versöhnung mit den Sirenen?}

Der Mensch ist, wie theateranthropologisch schon von Aristoteles in seiner Poetik erkannt, das nachahmende Tier. Menschen haben Freude an der ästhetischen (also im Realen folgenlosen) Nachahmung, so erkläre sich das Vergnügen am Theater. Übertragen auf das Schauspiel der Sirenen vor dem gefesselten Odysseus könnte man mit HORKHEIMER und ADORNO argumentieren: „Nachahmung tritt in den Dienst der Herrschaft, indem noch der Mensch vorm Menschen zum Anthropomorphismus wird. Das Schema der odysseischen List ist Naturbeherrschung durch solche Angleichung“" (HORKHEIMER / ADORNO 1968:74). Anthropomorphismus, so könnte daraus gefolgert werden, wäre die Grundlage der Entwicklung des Dramas und der neuzeitlichen Dramaturgie seit der griechischen Antike. Dramatisches Theater, ja im Grunde jede dramatische Mediendramaturgie (wie heute etwa die TV-Serie, der postklassische Film etc.) wären Projektionen des intelligenten Odysseus, des reflektierenden, sich selbst bewussten Menschen, der die Begegnung mit den Sirenen genießen, aber auch als stabiles Selbst überstehen will. Das funktioniert für HORKHEIMER und ADORNO nur um den Preis der Entsagung, der listige Mensch ,überlebe nur um den Preis des eigenen Traums, den er abdingt, indem er ihn wie die Gewalten draußen sich selbst entzaubert" (HORKHEIMER / ADORNO 1968:74). Er winde ,sich durch, das ist sein Überleben, und aller Ruhm, den er selbst und die andern ihm dabei gewähren, bestätigt bloß, dass die Heroenwürde nur gewonnen wird, indem der Drang zum ganzen, allgemeinen, ungeteilten Glück sich demütigt“" (HORKHEIMER / ADORNO 1968:74). Die Reise des modernen Helden Odysseus begründet nicht allein die Entwicklung des Dramas seit Aischylos, Sophokles, Euripides und Aristophanes, sondern auch seit Ende des 19. Jahrhunderts die

$5 \quad$ Vgl. zur an der ontologischen Differenz orientierten mimetischen Differenz als mediale Spezifität des Theaters WALDENFELS (2004). 
klassische Filmerzählung: Joseph Campbells Held*innenreise, der Monomythos als populäre Dramaturgie, ermittelt in der vergleichenden Strukturanalyse von Erzählungen, Mythen und Märchen (vgl. CAMPBELL 2007). In dieser werden die filmischen Held*innen zum Abenteuer aufgefordert, brechen nach erster Weigerung mit der Hilfe von Mentor*innen dennoch auf, bewältigen Schwierigkeiten, widerstehen Versuchungen, meistern Prüfungen und kommen mit einem veränderten, höheren Bewusstsein wieder nach Hause zurück (vgl. KRÜTZEN 2004; CunNINGHAM 2008). Eine der Versuchungen - wenn man Campbells Monomythos mit HORKHEIMERS und ADORNOs Deutung korreliert - wären die Sirenen als Prüfung nur zu meistern für die Held*innen mit ihrem „Organ des Selbst, Abenteuer zu bestehen, sich wegzuwerfen, um sich zu behalten" (HORKHEIMER / ADORNO 1968:64), also der reflektierenden List (vgl. KRÜTZEN 2018). Auf institutioneller Ebene treten die Sirenen somit in den Dienst der gewinnorientierten Kulturindustrie, deren Gewalt in ,ihrer Einheit mit dem erzeugten Bedürfnis liegt, nicht im einfachen Gegensatz zu ihm“ (HoRKHEIMER / ADORNO 1968:163). Gegen die Option der List zur Erhaltung der sich selbst bewussten eigenen Identität mittels der medialen Angebote der Kulturindustrie stünde eine annähernd in die gegenteilige Richtung zielende ästhetische Strategie der (Neo-)Avantgarde, die mehr oder weniger bedingungslose Übereignung des Selbst an die Sirenen. Eine solche Auflösung im Dionysischen (Nietzsche), im Theater des Lebens als Theater der Grausamkeit (Artaud) erlaubt gegebenenfalls eine Wiederverzauberung der Welt in einer Ästhetik des Performativen. ${ }^{6}$ Beides, die bürgerliche Bindung der Kraft der Sirenen an den dramatischen Dialog des kommerziellen Films oder der populären TV-Serie auf der einen Seite wie auch der performative Rausch in der Verzauberung einer grenzüberschreitenden Performance auf der anderen Seite, wäre wohl nicht nach ADORNOS Geschmack gewesen. Die Avantgarde mit Abstand und theoretisch reflektiert genießen, zugleich aber den Reflexionsraum des Hörsaals von den körperlichen Überwältigungen der nackten Provokation der Studentinnen freizuhalten, wäre eher im Sinn des Professors und Philosophen gewesen. Nicht das grausame Reale, sondern die Kunst habe, so ADORNO in seiner Ästhetischen Theorie, „das Unversöhnliche zu bezeugen und gleichwohl tendenziell zu versöhnen; möglich ist ihr das nur in ihrer nicht-diskursiven Sprache“ (ADORNO 1973:251). Dem Ästhetischen gelänge sozusagen symbolisch, was den Diskursen

So die These von FISCHER-Lichte (2004:362). 
versagt blieb. ${ }^{7}$ Es scheint sich aus dem zu speisen, was Alexander Kluge unter dem Urvertrauen des Menschen versteht (vgl. KLUGE 2004). Daher habe das Ästhetische dialektisch zu versöhnen, nicht energetisch - schon gar nicht roh körperlich - aufzubrechen. Aktuellstes deutschsprachiges Theater in diesem Sinne präsentiert Simon Stone etwa durch seine neorealistischen Überschreibungen, die sich erkennbar an der Dialogstruktur von TV-Qualitätsserien orientieren und dennoch theatrale Präsenz behaupten. Christopher Rüping versetzt die Tetralogie der Tragödien und des Satyrspiels in Dionysos Stadt (die in der Umfrage von Theater heute 2019 zur Inszenierung des Jahres gewählt wurde) aus dem Dionyostheater Athens zehn Stunden lang in das Stadttheater der Münchner Kammerspiele, wobei sich die Orestie des Aischylos zur Familiensoap und Schlagerparade wandelt und die Macht der Sirenen ihr Ventil in Zinédine Zidanes unbegreifbaren Kopfstoß im WM-Finale von Berlin findet. Auch auf den leitenden Ebenen spielt der Geist ADORNOS keine Nebenrolle: Für Zürich gilt nun mit Benjamin von Blomberg und Nicolas Stemann: „Theater besteht darin, Gegensätze zu denken“. Andreas Beck beginnt am Münchner Residenztheater die Spielzeit mit der leitenden Frage: „Was ist (uns) der Mensch?“8 Nichts Neues also im alten Kunstmedium des Theaters. Um den sich selbst bewussten, dennoch zerrissenen, in sich uneinigen Menschen ging es bereits Homer: Odysseus, gebunden am Mast, verzückt und leidend den auf Abstand gehaltenen Sirenen lauschend, so seinen Gegensätzen bewusst, jedoch seine Identität tapfer erkundend, erspielend und prägnant gestaltend - das scheint seit jeher und in Zukunft ein treffendes Bild für das Theater und die darstellenden wie performativen Künste zu sein.

7 Vielleicht wären die Sirenen als Naturgottheiten im Moment noch mal ganz anders zu interpretieren - als Moment des Erschreckens einer sich wehrenden Natur, wie es die protestierenden Schüler*innen artikulieren, die sich seit einem Jahr zunehmend in den Aktionen Fridays for Future engagieren. In diesem Sinne wäre gegebenenfalls der von HORKHEIMER und ADORNO eingeführte Begriff der instrumentellen Vernunft hilfreich, den gesellschaftlich-politischen Hintergrund des Klimawandels zu beleuchten.

8 Vgl. hierzu die jeweiligen Ankündigungen der neuen Spielzeit 19/20 des Schauspielhauses Zürich (https://www.zkb.ch/de/uu/sp/kultur-szene/theater/schauspielhaus (01.10.2019) und des Münchner Residenztheaters (https://www.residenztheater.de/das-haus (01.10.2019). 
Der performative Zauber der Sirenen? Adornos negative Dialektik...

\section{Literatur}

Adorno, THEODOR W. (1970): Negative Dialektik. Frankfurt a. M.

Adorno, Theodor W. (1973): Ästhetische Theorie. Frankfurt a. M.

Adorno, THeOdor W. (1994): Brief vom 18. März 1936 an Walter Benjamin. In: AdORnO, Theodor W. / Benjamin, WALter: Briefwechsel. Bd. 1: 1928-1940. Frankfurt a. M., 168-175.

Adorno, TheOdor W. (2005): Traumprotokolle, ed. GÖDDE, Christoph; LONITZ, HENRI. Frankfurt a. M.

Betram, GeOrG W. (2005): Kunst. Stuttgart.

BoenM, GOTTFRIED (2003): Der Topos des Lebendigen. Bildgeschichte und ästhetische Erfahrung. In: KÜPPER, JOACHIM / MENKE, CHRISTOPH (eds.): Dimensionen ästhetischer Erfahrung. Frankfurt a. M., 203-222.

BÜRger, PETER (1974): Theorie der Avantgarde. Frankfurt a. M.

CAmpbell, Joseph (1949): The Hero with a Thousand Faces. New York.

Cunningham, KeIth (2008): The Soul of Screenwriting. On Writing, Dramatic Truth, and Knowing Yourself. New York.

FisCHER-LiCHTE, ERIKA (2004): Ästhetik des Performativen. Frankfurt a. M.

Homer (1977): Die Odyssee. Übers. von Johann Heinrich Voss. Stuttgart.

Horkheimer, Max / Adorno, Theodor W. (1968): Dialektik der Aufklärung. Philosophische Fragmente. Amsterdam.

Kluge, Alexander (2004): Chronik der Gefühle. Frankfurt a. M.

KrÜtzen, Michaela (2004): Dramaturgie des Films. Frankfurt a. M.

Krützen, Michaela (2018): Dramaturgie des Films. In: Englhart, Andreas / SCHÖßLER, FRANZISKA (eds.): Grundthemen der Literaturwissenschaft: Drama. Berlin, 557-576.

Lehmann, Hans-Thies (1999): Postdramatisches Theater. Frankfurt a. M.

LEMHÖFER, ANNE (2008): Busenattentat im Raum für Ideen: https://www.fr.de/politik/ busenattentat-raum-ideen-11592206.html (01.11.2019).

MenKe, Christoph (2013): Die Kraft der Kunst. Berlin.

MERSCH, DIETER (2001): Aisthetik und Responsivität. Zum Verhältnis von medialer und amedialer Wahrnehmung. In: FISCHER-LICHTE, ERIKA u.a. (eds.): Wahrnehmung und Medialität. Tübingen, 273-300.

NiETzSCHE, FRIEDRICH (1988): Die Geburt der Tragödie. In: Ders.: Kritische Studienausgabe. Bd. 1, ed. Colli, Giorgio; Montinari, Mazzino. München, 9-156.

OSTERMEIER, THOMAs (2009): Erkenntnisse über die Wirklichkeit des menschlichen Miteinanders. Plädoyer für ein realistisches Theater: http://www.schaubuehne.de/uploads/Realistisches-Theater.pdf (01.11.2019). 
Piketty, Thomas (2014): Capital in the Twenty-First Century. Cambridge / Massachusetts. POLLESCH, RENÉ (2010): Ich schau dir in die Augen, gesellschaftlicher Verblendungszusammenhang! UA: 13. Jan. 2010, Volksbühne am Rosa-Luxemburg-Platz, Berlin; Regie: René Pollesch (Text unveröffentlicht).

Rimini PRotóoll (2006): Interview. Archiv der Berliner Festspiele 2006: http:// archiv2berlinerfestspiele.de/de_1/archiv/festivals2006/03_theatertreffen06/tt_06_forum/tt_06_forumprogramm/tt_06_forumworkshop1.php (01.11.2019).

SCHECHNER, RICHARD (1966): Approaches to Theory / Criticism. In: The Tulane Drama Review 4:20-53.

SCHECHNER, RiCHARD (1973): Environmental Theatre. New York.

SCHECHNER, RichaRd (1977): Essays on Performance Theory. New York.

SeEl, Martin (2007): Die Macht des Erscheinens. Frankfurt a. M.

Stegemann, BeRnd (2015): Kritik des Theaters. Berlin.

TRAuB, UlRike (2010): Theater der Nacktheit. Zum Bedeutungswandel entblößter Körper auf der Bühne seit 1900. Bielefeld.

Vogler, Christopher (2007): The Writer's Journey. Mythic Structure for Writers. Studio City.

Waldenfels, Bernhard (2004): Mimetische Differenz und pathische Impulse. In: Rimavesi, PATRICK u.a. (eds.): AufBrüche. Theaterarbeit zwischen Text und Situation. Festschrift für Hans-Thies Lehmann. Berlin, 62-67.

WIRTH, ANDRZEJ (1985): Säkularisierung des deutschen Theaters. In: Gießener Universitätsblätter 181: 45-48: http://geb.uni-giessen.de/geb/volltexte/2013/9606/ (12.09.2018). 